\title{
Improvement of ORB algorithm
}

\author{
Xue Leng ${ }^{\text {a }}$, Jinhua Yang
}

School of Photoelectric Engineering, Changchun University of Science and Technology, Changchun, 130000, China

aLengxue79@126.com.

Keywords: ORB, Improved ORB, Scale invariance.

\begin{abstract}
In this paper, According to the idea SIFT algorithm, proposed an improved algorithm ORB Based on the multi-scale space, detecting scale space extreme points, then use the ORB descriptors to describe the feature points, generated descriptors having rotating invariability. Using Hamming distance to achieve matching feature points. Achieved good results.
\end{abstract}

\section{ORB feature points matching algorithm}

ORB algorithm using corner detector to extract the feature points, corner point is defined as: there are plenty of pixels with a point in different regions in the neighborhood around the pixels. In the feature point centered $\mathrm{D}$ compare the size between the gray value, the center of the gray values of $\mathrm{D}$ and all points on the gray value to a circle centered at point $\mathrm{D}$. When the number of points of $\mathrm{D}$ point on the circumference of the gray value difference is large enough, considered as a feature point candidate point D. Corner detected feature points do not have the scale invariance and rotational invariance. In order to have a rotation invariant of ORB descriptor, need to assign a direction for each feature point. Therefore in the centere of feature point, in its neighborhood calculated the centroid position. Then the feature points as a starting point to construct a vector for the end of the centroid. This direction of vector is the direction of the feature point. Calculated as follows:

$M_{p, q}=\sum_{(x, y) \in S} x^{p} y^{q} f(x, y)$

$\mathrm{f}(\mathrm{x}, \mathrm{y})$ represents the gray values of the image, the centroid position of this area is:

$C=\left(\frac{M_{1,0}}{M_{0,0}}, \frac{M_{0,1}}{M_{0,0}}\right)$

Feature point direction is:

$\theta=\arctan \left(M_{0,1} / M_{1,0}\right)$

\section{ORB feature point Descriptor}

ORB rotation invariant Descriptor is a form of binary code string descriptor, the idea is the neighborhood of image feature points can be used to express a small amount of gray contrast its calculation is simple and fast. Definition the test criteria of the image neighborhood:

$$
\tau(p ; x, y)=\left\{\begin{array}{l}
1, p(x)<p(y) \\
0, p(x) \geq p(y)
\end{array}\right.
$$

$p(x)$ is the gray value, select $\mathrm{n}$ test points, defined binary criteria, such descriptor isn-dimensional binary code strings.

$$
f_{n}(p)=\sum_{1 \leq i \leq n} 2^{i-1} \tau\left(p ; x_{i}, y_{i}\right)
$$

n may be 128,256 or 512required between the computation, storage spaces and the recognition rate tradeoff. Descriptor itself is no direction, does not have rotational invariance, use of feature points obtained centroid direction, such that the direction information Descriptor contains feature point. At the point (x, y), define a 2 xn matrix: 


$$
Q=\left(\begin{array}{l}
x_{1}, x_{2}, \ldots x_{n} \\
y_{1}, y_{2}, \ldots y_{n}
\end{array}\right)
$$

$\left(x_{i}, y_{i}\right)$ represents a test points, Use the feature points corresponding to the direction rotating matrix, correction matrix can be constructed. So that you can get having rotating invariability Descriptor.

$$
g_{n}(p, \theta)=f_{n}(p) \mid\left(x_{i}, y_{i}\right) \in Q_{\theta}
$$

\section{SIRB algorithm}

Use of multi-scale space to extract the features with scale invariance

Point in SIFT algorithm, then describe the feature points ORB, generated ORB Descriptor, using Hamming distance matching feature points. Custom image $F(x, y)$ scale is $L(x, y, \sigma), \sigma$ isscale factor , it is obtained from the Gaussian function and image convolution.

$$
\begin{aligned}
& L(x, y, \sigma)=G(x, y, \sigma) F(x, y) \\
& G(x, y, \sigma)=\frac{1}{2 \pi \sigma^{2}} \exp \left(-\frac{x^{2}+y^{2}}{2 \sigma^{2}}\right)
\end{aligned}
$$

In order to effectively detect scale space stable feature points, find the extreme points in space of difference of Gaussian function and image convolution, local extreme point scale space as a candidate feature points.

$$
\begin{aligned}
& D(x, y, \sigma)=[G(x, y, k \sigma)-G(x, y, \sigma)] F(x, y) \\
& =L(x, y, k \sigma)-L(x, y, \sigma)
\end{aligned}
$$

After seek extreme points, need to be screened for extreme points, remove the unstable point. In an extreme points, Taylor expansion for $D(x, y, \sigma)$.

$$
D(x)=D+\frac{\partial D^{T}}{\partial X} X+\frac{1}{2} X^{T} \frac{\partial^{2} D}{\partial X^{2}} X
$$

Obtained partial derivatives of $X$, order partial derivatives to zero, you can get extreme points.

$$
\bar{X}=-\frac{\partial^{2} D^{-1}}{\partial X^{2}} \frac{\partial D}{\partial X}
$$

Put (11) into (10)

$$
D(\bar{X})=D+\frac{1}{2} \frac{\partial D^{T}}{\partial X} \bar{X}
$$

If the above formulas is less than 0.03 , then remove the corresponding extreme points. In order to obtain a stable extreme points, also need to remove the extreme points on the edge. Heather matrix is:

$$
H\left(\begin{array}{l}
D_{x x} D_{x y} \\
D_{x y} D_{y y}
\end{array}\right)
$$

Let $\alpha$ is the largest eigenvalue of $H, \beta$ is the smallest eigenvalue. From the nature of the matrix

$$
\left\{\begin{array}{l}
\operatorname{Trace}(H)=D_{x x}+D_{y y}=\alpha+\beta \\
\operatorname{Det}(H)=D_{x x} D_{y y}-\left(D_{x y}{ }^{2}\right)=\alpha \beta
\end{array}\right.
$$

Eigenvalue of $H$ is proportional to the curvature of the main of $D$, so just concerned about the ratio of eigenvalues. Let $\mathrm{Q}$ is the ratio of the maximum and minimum eigenvalues. It can be obtained:

$$
\frac{\operatorname{Trace}(H)^{2}}{\operatorname{Dec}(H)}=\frac{(\alpha+\beta)^{2}}{\alpha \beta}=\frac{(\gamma \beta+\beta)^{2}}{\gamma \beta^{2}}=\frac{(\gamma+1)^{2}}{\gamma}
$$

To check whether the ratio of principal curvature less than the threshold value, only need to check whether the establishment of the following formula. 


$$
\frac{\operatorname{Trace}(H)^{2}}{\operatorname{Dec}(H)}<\frac{(\gamma+1)^{2}}{\gamma}
$$

Usually the threshold value is8, believe that the main curvature ratio greater than 8 extreme points of the point on the edge, thus be cleared.

\section{4. experimental results}

We did a survey with matching accuracy of SIRE and ORB. As shown in Table 1.

Table 1

\begin{tabular}{ccc}
\hline Groups & \multicolumn{2}{c}{ Matching Algorithm } \\
\cline { 2 - 3 } & SIRB & ORB \\
\cline { 2 - 3 } & & \\
1 & 94.6 & 24.3 \\
2 & 94.8 & 28.6 \\
3 & 95.6 & 29.7 \\
4 & 90.2 & 16.8 \\
5 & 91.3 & 19.6 \\
6 & 92.6 & 26.5 \\
7 & 93.5 & 17.9. \\
8 & 89.6 & 15.8 \\
Average & 92.8 & 22.4 \\
\hline
\end{tabular}

As can be seen from Table 1, when the image scale changes, SIRB feature point matching accuracy is much higher than the accuracy of the algorithm matches ORB. Matching accuracy SIRB average about 92.8 , increased by about $70 \%$ than the matching accuracy ORB, description SIRB algorithm superiority on the scale invariability. Similarly, we do comparative experiments of match time. The results shown in Table 2.

\begin{tabular}{ccc}
\multicolumn{3}{c}{ Table 2 } \\
\hline Groups & \multicolumn{3}{c}{ Matching Algorithm } \\
\cline { 2 - 3 } & & ORB \\
& & \\
\cline { 2 - 3 } & & \\
1 & 47.6 & 35.6 \\
2 & 42.3 & 30.2 \\
3 & 52.6 & 25.4 \\
4 & 46.2 & 19.8 \\
5 & 56.1 & 32.3 \\
6 & 48.6 & 27.5 \\
7 & 50.5 & 28.6 \\
8 & 36.8 & 34.2 \\
Average & 47.5 & 32.6 \\
\hline
\end{tabular}

From the table 2, we can see that SIRB average match time is about $47.5 \mathrm{~ms}$, and ORB average match time is $32.6 \mathrm{~ms}$. Two algorithms to match approximately the same time.

\section{Summary}

Improved algorithm effectively solves the ORB defects, retains the advantages rapid of ORB. When the image scale changes, SIRB can accurately match the feature points. The average increase of $70 \%$ matching accuracy than ORB, the average match time is almost equal with the ORB. 


\section{References}

[1] WANG K SH. Summarize of local invariant feature. Chinese Journal of Scientific Instrument. VOL. 2 (2011) NO.2, P. 141-151.

[2] Zhang K Y, Liang F M. Research on the key algorithm for image matching based on improved SURF.Science Technology and Engineering, VOL. 10(2013) NO. 13, P: 2875-2879.

[3] Fan Z Q, Zhao Q P. A data-clustering based robust SIFT feature matching method, Journal of Computer Research and Development. VOL. 5(2012) NO. 49, P: 1123-1129.

[4] Tang C W, Xiao J, Shao Y Q, et al. Global structured SIFT descriptor and its application in image matching.J Huazhong Univ of Sei \&Tech (Natural Science Edition), VOL.1 (2012) NO.40, P: 15-20.

[5] Du J Y, Hu H M,Liu Y C. Research and implementation of matching technique of image based on SIFT algorithm of area blocking Opto-electronic Engineering,2VOL.8(2013)NO.:40,P:52-58. 